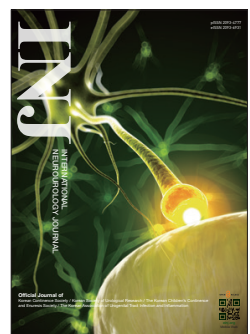

\title{
Frontiers in the Clinical Applications of Botulinum Toxin A as Treatment for Neurogenic Lower Urinary Tract Dysfunction
}

\author{
Yuan-Hong Jiang, Sheng-Fu Chen, Hann-Chorng Kuo \\ Department of Urology, Hualien Tzu Chi Hospital, Buddhist Tzu Chi Medical Foundation, Tzu Chi University, Hualien, Taiwan
}

\begin{abstract}
Patients with neurogenic lower urinary tract dysfunction (NLUTD) experience urinary incontinence with or without difficult urination, which might promote recurrent urinary tract infection (UTI) and exacerbate upper urinary tract function. Nonetheless, appropriate bladder management has been shown to reduce urological complications and improve quality of life. In addition to pharmacological therapy and surgical intervention, botulinum toxin A (BoNT-A) has been widely utilized in NLUTD. The therapeutic efficacy of detrusor BoNT-A injections for neurogenic detrusor overactivity due to spinal cord injury (SCI), multiple sclerosis, or other central nervous system lesions, such as cerebrovascular accident, Parkinson disease, early dementia, and pediatric NLUTD due to myelomeningocele, has been well established, with repeated BoNT-A injections every 6 to 9 months being necessary to maintain its therapeutic effects. Urethral BoNT-A injection can decrease urethral sphincter resistance and facilitate efficient voiding in patients with NLUTD who wish to preserve self-voiding. Detrusor BoNT-A injection can also decrease the occurrence of autonomic dysreflexia in patients with SCI, even after failed augmentation enterocystoplasty, with additional benefits including reduced UTI episodes and preserved renal function with repeated injections. However, this treatment does have some side effects. Complete informed consent for BoNT-A injection therapy with full disclosure of its potential complications should therefore be obtained before this procedure is undertaken.
\end{abstract}

Keywords: Botulinum toxins; Urinary bladder, Neurogenic; Detrusor; Urinary incontinence; Urodynamics

- Conflict of Interest: No potential conflict of interest relevant to this article was reported.

\section{INTRODUCTION}

Neurogenic lower urinary tract dysfunction (NLUTD) causes various lower urinary tract dysfunctions following neurological diseases or injuries. Accordingly, patients may experience urine storage failure due to detrusor overactivity (DO) or urethral sphincter incompetence; bladder emptying failure due to detrusor areflexia, detrusor underactivity, bladder neck dysfunction, or detrusor external sphincter dyssynergia (DSD); or combined urine storage and emptying failure due to DSD or detrusor hyperreflexia and inadequate contractility. Botulinum toxin A (BoNT-A), which was initially utilized in urology as a treat- ment for patients with NLUTD due to chronic spinal cord injuries (SCIs) [1-4], selectively modulates neurotransmission from motor nerve endings to cause muscular paralysis, acts on sensory receptors to decrease pain and sensory disorders, and promotes anti-inflammatory effects to improve chronic inflammation and related functional disorders. Moreover, studies have shown that BoNT-A injection into the urethral sphincter improves voiding efficiency in patients with DSD [5], while others have revealed that detrusor BoNT-A injection reduces DO and intravesical pressure and improves urinary incontinence [6,7]. The clinical applications of BoNT-A can be further extended to include the treatment of neurogenic DO (NDO) and urinary

Corresponding author: Hann-Chorng Kuo (iD https://orcid.org/0000-0001-7165-4771 Department of Urology, Hualien Tzu Chi Hospital, Buddhist Tzu Chi Medical Foundation, 707, Section 3, Chung-Yang Road, Hualien, Taiwan E-mail:hck@tzuchi.com.tw

Submitted: September 29, 2020 / Accepted after revision: October 21, 2020 
Table 1. Clinical applications of botulinum toxin A for neurogenic lower urinary tract dysfunction

\begin{tabular}{llccc}
\hline NLUTD & \multicolumn{1}{c}{ Therapeutic effects } & Target & Usual dose & References \\
\hline SCI, DSD & Improved self-voiding, decreased PVR & Urethra & $100 \mathrm{U}$ & $1-5,18,19,22,27$ \\
SCI, NDO & Improved continence, decreased detrusor pressure, & Detrusor & $200-300 \mathrm{U}$ & $6,7,16,20,23,39,40,42$, \\
& increased bladder capacity, protected upper tract & & $43,46,47,49,53,54,55$ \\
SCI, AD & Decreased AD & Detrusor & $200 \mathrm{U}$ & 28,30 \\
MS, PD, CVA, NDO & Improved continence, decreased detrusor pressure, & Detrusor & $200-300 \mathrm{U}$ & $59,60,61,65,67,62,63$, \\
& increased bladder capacity, preserved self-voiding & Detrusor & $100 \mathrm{U}$ & $64,66,68$ \\
MS, PD, CVA, DSD & Improved self-voiding, decreased PVR & Urethra & $100 \mathrm{U}$ & 5 \\
Pediatric MMC, NDO & Increased bladder capacity, decreased detrusor & Detrusor & $5-10 \mathrm{U} / \mathrm{kg}$ & $73,74,75$ \\
& pressure, increased compliance, decreased VUR & & Total 10 U/kg & 78,80
\end{tabular}

NLUTD, neurogenic lower urinary tract dysfunction; SCI, spinal cord injury; DSD, detrusor sphincter dyssynergia; NDO, neurogenic detrusor overactivity; AD, autonomic dysreflexia; MS, multiple sclerosis; PD, Parkinson disease; CVA, cerebrovascular accident; PVR, postvoid residual; VUR, vesicoureteral reflux; MMC, myelomeningocele.

incontinence among pediatric patients with myelomeningocele and other types of NLUTD, as well as autonomic dysreflexia (AD) in patients with high-level SCI (Table 1). However, urinary tract infection (UTI) remains the most bothersome adverse event (AE) prohibiting its widespread use in actual clinical practice. The current review focuses on the frontiers in the clinical applications of BoNT-A for the treatment of NLUTD.

\section{MECHANISM OF ACTION OF BOTULINUM TOXIN A FOR NEUROGENIC LOWER URINARY TRACT DYSFUNCTION}

BoNT-A has been found to cleave N-ethylmaleimide-sensitive fusion attachment protein receptor complex (SNAP-25), thereby inhibiting neurotransmitter release at the neuromuscular and neuroglandular junctions [8]. The pharmacological mechanism of action of BoNT-A for lower urinary tract dysfunction in humans may involve not only efferent function, but also afferent nerve terminals and the urothelium [9-11].

Studies have shown that the detrusor injection of BoNT-A affects preganglionic parasympathetic and adrenergic nerves and sensory fibers, blocking acetylcholine ( $\mathrm{ACh})$ release and promoting paralysis of bladder motor neurons $[9,10]$. Moreover, other studies have found a reduction in human bladder muscarinic receptors $\mathrm{M} 2$ and $\mathrm{M} 3$ and purinergic receptors $\mathrm{P} 2 \mathrm{X} 2$ and P2X3 after detrusor BoNT-A injections [12]. By inhibiting both sensory and motor nerves in the micturition reflex, BoNT-A injections can attenuate DO in the bladders of patients with SCI. BoNT-A has also been found to alter the re- lease of adenosine triphosphate, neurotrophins, and nitric oxide (NO) in the urothelium, which can also reduce sensory urgency both in neurogenic and nonneurogenic bladder dysfunction [9,13-15]. One study showed that urethral sphincter BoNT-A injection can decrease urethral striated muscle tonicity, lower urethral resistance, and improve voiding efficiency in patients with detrusor sphincter dyssynergia [5]. Notably, BoNT-A has been found to have both motor and sensory inhibitory effects. However, considering that sensory inhibition is unrelated to ACh release, BoNT-A may have longer inhibitory effects on detrusor contractility than on the urethral sphincter $[2,16]$.

\section{URETHRAL SPHINCTER BOTULINUM TOXIN A INJECTION FOR DETRUSOR EXTERNAL SPHINCTER DYSSYNERGIA}

Urethral sphincter BoNT-A injection was initially used to treat DSD in patients with SCI to facilitate spontaneous urination without self-catheterization [1]. The therapeutic effect of urethral sphincter BoNT-A injection, which is typically dosed at $100 \mathrm{U}$, usually appears 1 week after injection and lasts for 3 to 6 months $[1-5,17,18]$. Accordingly, a decrease in postvoid residual (PVR) volume and maximal urethral closure pressure and an increase in maximum flow rate have been observed after BoNT-A injection [1,2]. Regardless of whether injections are performed transperineally or transurethrally, previous studies have confirmed treatment efficacy [3,4]. Notably, a $50 \%$ decrease in UTIs in NDO has been observed after BoNT-A injection, mainly due to the decrease in PVR and intravesical pres- 
sure following treatment [18]. However, urethral sphincter BoNT-A injection has yet to be widely utilized among patients with SCI considering the emerging evidence on AEs after injection, which include increased urinary incontinence and persistent incomplete bladder emptying [5,19-21]. The exacerbation of urinary incontinence in patients with SCI generally prohibits the clinical use of urethral BoNT-A injections in treating DSD. As such, patients are usually switched to detrusor BoNT-A injections to promote urinary continence despite the need for clean intermittent self-catheterization (CISC) [19,22,23].

Although urethral BoNT-A injections have not been widely utilized for DSD treatment in patients with SCI, this treatment may likely benefit those who have neurogenic lesions and discoordinated urethral sphincter during urination, as well as those who are ambulatory and prefer to urinate voluntarily. Previous studies have revealed that 100- to 200-U injections of urethral sphincter BoNT-A were effective for patients with multiple sclerosis (MS), cerebrovascular accident (CVA), or SCI $[5,20]$. Meanwhile, a study showed that clean intermittent catheterization (CIC) can be avoided in patients with CVA and chronic urinary retention after urethral BoNT-A injection of $100 \mathrm{U}$ [24]. However, repeat injections are necessary to maintain therapeutic efficacy.

\section{CURRENT ROLE OF BOTULINUM TOXIN A IN AUTONOMIC DYSREFLEXIA AMONG PATIENTS WITH SPINAL CORD INJURY}

Patients with SCI who have high-level spinal cord lesions typically present with $\mathrm{AD}$, a potentially life-threatening urological complication that results in hypertension, headaches, and even stroke when not treated appropriately [25]. AD is believed to be triggered by cutaneous or visceral afferent stimuli that originate below the level of the spinal cord lesion [26] and occurs most often in individuals with SCI above the T6 level [25]. Patients with chronic SCI may develop AD during bladder overdistention, stool impaction, or UTI [27]. Accordingly, studies have shown that BoNT-A injections into the detrusor or urethral sphincter abolished $\mathrm{AD}$ or decreased its severity in some patients with SCI $[6,27]$ perhaps by decreasing intravesical pressure or inhibiting sensory afferent pathways. Our previous study demonstrated that $\mathrm{AD}$ may resolve, persist, or exacerbate after detrusor BoNT-A injections in patients with SCI who had NDO. Overall, $62 \%$ of patients exhibited improvement in $\mathrm{AD}$ severity after BoNT-A injections, whereas only 10 patients de- veloped $\mathrm{AD}$ exacerbation after detrusor BoNT-A injections.

Patients with persistent $\mathrm{AD}$ after augmentation enterocystoplasty may also benefit from BoNT-A injections into the native bladder [28]. Although intravesical BoNT-A injection has been considered as a novel treatment to control $\mathrm{AD}$ in patients with high-level SCI, some may develop an acute $\mathrm{AD}$ response during detrusor BoNT-A injection. This is because detrusor BoNTA injection causes bladder wall trauma, acute suburothelial nerve plexus inflammation, and autonomic nervous system activation, thereby exacerbating $\mathrm{AD}$ during the acute stage of BoNT-A injection. This discomforting experience often discourages patients from undergoing repeat BoNT-A injections for NDO and urinary incontinence [29,30]. Nevertheless, detrusor BoNT-A injections should be attempted in patients with high-level SCI whose $\mathrm{AD}$ is refractory to conventional medical treatment in order to alleviate $\mathrm{AD}$ severity and improve quality of life.

\section{BOTULINUM TOXIN A FOR NEUROGENIC DETRUSOR OVERACTIVITY}

Less invasive and reversible treatment strategies, such as neurotoxin injection into the detrusor or urethral sphincter, should be utilized for bladder control and emptying in patients with NLUTD. Based on an understanding of its pharmacological mechanism, BoNT-A was enthusiastically utilized for the treatment of NDO by 2000. Intradetrusor BoNT-A injection has been demonstrated to be effective in restoring urinary continence for over 3 to 9 months [16,31,32]. Moreover, a BoNT-A dose of 200 to $300 \mathrm{U}$ injected into the detrusor successfully increased the cystometric bladder capacity, decreased voiding detrusor pressure, and improved urinary continence in patients with SCI. However, CIC remains mandatory for periodic emptying of the bladder [6]. Other studies have noted that this treatment decreased $\mathrm{AD}$ and significantly improved quality of life among patients with who had DSD [27,33].

Earlier studies found that a BoNT-A dose of $300 \mathrm{U}$ increased cystometric bladder capacity, decreased reflex volume, decreased detrusor voiding pressure, and increased bladder compliance [34-38]. Further clinical trials all confirmed that detrusor BoNT-A injection had superior therapeutic efficacy compared to placebo in patients with SCI or MS [16]. One study showed that a BoNT-A dose of 200 and $300 \mathrm{U}$ had similar therapeutic effects on NDO, and a dose of $200 \mathrm{U}$ was approved by the U.S. Food and Drug Administration (FDA) [39]. Moreover, 
repeated detrusor BoNT-A injections seem to be as effective as the initial injection [40]. A pooled data analysis of the effects of detrusor BoNT-A injection on NDO in patients with SCI found a $63 \%$ decrease in daily incontinence, an $18 \%$ decrease in CIC episodes, a $68 \%$ increase in cystometric bladder capacity, a $61 \%$ increase in reflex volume, and a $42 \%$ decrease in maximal detrusor pressure [41]. A further analysis of treatment outcomes also revealed significant improvements in health-related quality of life indices $[38,42]$ and a significant decrease in the incidence of symptomatic UTI after detrusor BoNT-A injections in patients with SCI [43]. Although AEs, such as UTI, bladder pain, temporary hematuria, and $\mathrm{AD}$, can occur after BoNT-A injection, the satisfaction rate of BoNT-A therapy has remained high in patients with SCI [44]. Considering the high incidence of UTI after BoNT-A injection, prophylactic antibiotic treatment is recommended to prevent symptomatic UTI after injection [45].

Concomitant use of anticholinergics, SCI etiology, or SCI levels did not affect the therapeutic effects of BoNT-A on NDO due to SCI or MS [46,47]. Patients with SCI who received 200 $\mathrm{U}$ detrusor injections of BoNT-A for NDO exhibited significantly greater satisfaction in terms of improvements in urinary incontinence and quality of life than those who received injections of $100 \mathrm{U}$ of BoNT-A into the urethral sphincter to improve bladder emptying [23].

To maintain the therapeutic efficacy of BoNT-A for NDO, repeated detrusor BoNT-A injections are mandatory. Among patients with SCI who received BoNT-A injections for NDO, $67 \%$ continued to receive repeated BoNT-A injections, of whom $90 \%$ had a high level of satisfaction and considered longterm BoNT-A treatment over the following 5 years [48]. Clinically, detrusor injections of both 200 and $300 \mathrm{U}$ of BoNT-A promoted greater improvement in subjective patient satisfaction compared to placebo [49].

Several open-label studies have shown that detrusor BoNT-A injections improved clinical and urodynamic parameters and quality of life in patients with refractory NDO $[7,16,50]$, while our previous study revealed that a single BoNT-A injection for NDO in SCI patients had a $73.3 \%$ success rate [51]. Furthermore, several phase 3 placebo-controlled multi-center clinical trials investigating the efficacy and safety of detrusor BoNT-A injections in patients with NDO due to SCI or MS revealed that 200 or $300 \mathrm{U}$ of BoNT-A promoted significantly fewer urinary incontinence episodes compared to placebo. Moreover, the therapeutic efficacy of BoNT-A injections was similar in both
SCI and MS [39,52,53]. After BoNT-A treatment, $62.9 \%$ and $61.6 \%$ of patients in the 200 and $300 \mathrm{U}$ groups achieved the subjective primary treatment goal of dryness, respectively [54]. Given the similar therapeutic efficacy, the regulatory dose of BoNT-A approved by the FDA of the United States, European Union, and Asian countries was 200 U for SCI or MS patients with NDO. However, in actual clinical practice, patients with SCI who received injections of $300 \mathrm{U}$ of BoNT-A for NDO had lower detrusor pressure and fewer involuntary detrusor contractions (IDCs) than those receiving $200 \mathrm{U}$ (44.1\% vs. 89.5\%, $\mathrm{P}=0.001$ ) during repeated injections [55]. The lower incidence of IDCs in the $300 \mathrm{U}$ group than in the $200 \mathrm{U}$ group after the second BoNT-A injection suggests that $300 \mathrm{U}$ of BoNT-A could potentially have a longer therapeutic duration than $200 \mathrm{U}$. Hence, patients who experienced a short therapeutic duration with $200 \mathrm{U}$ BoNT-A injection should be treated with $300 \mathrm{U}$ BoNT-A when the initial treatment effect disappears [55].

\section{ADVERSE EVENTS OF BOTULINUM TOXIN A INJECTIONS FOR NEUROGENIC BLADDER}

AEs related to BoNT-A injections have often been observed, with the most frequent being UTI and urinary retention requiring CIC [53]. Clinically, although detrusor BoNT-A injections effectively reduce urinary incontinence, they usually impair detrusor contractility, resulting in large PVR or urinary retention in NDO, with approximately $70 \%$ of patients requiring periodic $\mathrm{CIC}$, and subsequent UTIs as de novo problems [7]. Considering the unrealistic expectations that patients receiving BoNT-A injections for urinary incontinence or difficulty in urination might have regarding treatment, some might be disappointed by the de novo AEs or complications occurring after BoNT-A treatment, promoting low satisfaction rates. Accordingly, increased incontinence was found to be the leading cause of dissatisfaction with urethral BoNT-A injections, while increased PVR volume requiring CIC was found to be the primary cause of dissatisfaction with detrusor injections [56].

One study found that more than $60 \%$ of patients with NDO due to SCI or MS receiving 200 or $300 \mathrm{U}$ of BoNT-A injections had no IDCs by week 6 [35]. Apart from UTI and large PVR, detrusor BoNT-A injections were reported to cause de novo $\mathrm{AD}$ [52], which was related to the bladder injection, rather than to BoNT-A. Our previous study found that some patients with SCI developed de novo $\mathrm{AD}$ after detrusor onabotulinumtoxinA injections (23.7\% and $17.6 \%$ in the 200 and 300 U groups, re- 
spectively) [30,55]. De novo AD after onabotulinumtoxinA injection could have likely been triggered by noxious stimuli due to transient bladder wall inflammation after multiple detrusor injections.

The aforementioned intolerable AEs often discourage patients from repeat BoNT-A injections, despite the potential for achieving treatment goals after BoNT-A injection. Our small series of patients with SCI who received repeated BoNT-A injections for NDO reported improvements in the satisfaction rate from $25.4 \%$ at baseline to $74 \%$ at 3 months after the fourth injection, although a slight decrease was noted after the fourth injection [30]. The overall satisfaction rate after single or repeated injections was $59.3 \%$ (35 patients), the failure rate was $33.9 \%$ (20 patients), and discontinuation rate due to AEs (2 recurrent UTIs and 2 cases of $\mathrm{AD}$ ) was $6.8 \%$ (4 patients). Among the 20 patients who reported treatment failure, $10(16.9 \%)$ reported no significant improvement after 1 or repeated injections, 8 converted to augmentation enterocystoplasty, and only $20 \%$ of patients continued repeated treatment. Nevertheless, patients with NDO due to SCI who continued to receive detrusor BoNT-A injections exhibited sustained improvement in voided volume and quality of life with repeated treatment [34].

\section{BOTULINUM TOXIN A INJECTIONS FOR NEUROGENIC DETRUSOR OVERACTVITY DUE TO MULTIPLE SCLEROSIS, CEREBROVASCULAR ACCIDENT, PARKINSON DISEASE, AND DEMENTIA}

Patients with neurogenic lesions other than SCI, such as those with MS, CVA, Parkinson disease (PD), and early dementia, may also have DO and urinary incontinence. The incidence of overactive bladder $(\mathrm{OAB})$ symptoms increases with age among patients with central nervous system (CNS) lesions. Accordingly, the incidence of urinary incontinence varied from $33 \%$ to $79 \%$ in patients with CVA [57], 33.1\% in those with PD, and $50.9 \%$ in those with MS [58]. Most patients with CNS disorders and $\mathrm{OAB}$ exhibited NDO. Although such patients experience urinary incontinence due to NDO, they may still have ambulatory ability and bladder sensation. Therefore, spontaneous urination without CIC is usually requested when BoNT-A injection is suggested for the treatment of NDO.

Previously, a 200- or 300-U dose of BoNT-A was recommended for the treatment of urinary incontinence in patients with MS [59]. However, patients receiving the aforementioned
BoNT-A dose need CIC for bladder emptying, despite a continence rate of $76 \%$ after $300 \mathrm{U}$ of BoNT-A injection [60]. Patients with MS might experience progression of disability, resulting in increased difficulty in CIC and discontinuation of repeated BoNT-A injections [61]. As such, a small BoNT-A dose of $100 \mathrm{U}$ is recommended for patients with MS who wish to improve urinary incontinence and preserve bladder sensation and spontaneous voiding after treatment. Improvements in urodynamic and voiding diary variables have been noted after injecting $100 \mathrm{U}$ of BoNT-A [62]. However, a high BoNT-A dose of 200 or $300 \mathrm{U}$ followed by CIC would still be the treatment of choice to restore urinary continence among patients with severe disability and urinary incontinence.

For patients with urinary incontinence due to CNS lesions, such as CVA, PD, or early dementia, a BoNT-A dose of $100 \mathrm{U}$ can effectively decrease urgency symptoms and improve clinical and urodynamic parameters for more than 6 months [63, 64]. A previous study using detrusor injections of $200 \mathrm{U}$ of BoNT-A provided beneficial efficacy for patients with urinary incontinence due to PD and multiple system atrophy [65]. Another study confirmed that injecting $100 \mathrm{U}$ of BoNT-A into the bladder of patients with PD promoted therapeutic effects without urinary retention [66]. However, not all patients with CNS lesions have similar treatment outcomes. Given that patients with CVA might not perceive urinary urgency until full bladder capacity is reached, such patients may not achieve complete urinary continence after treatment with $200 \mathrm{U}$ of BoNT-A, with one study showing urodynamic improvements in only $50 \%$ of patients [67]. Our recent study also revealed the therapeutic efficacy and safety of $100 \mathrm{U}$ of BoNT-A bladder injections in elderly patients with chronic CVA, $\mathrm{PD}$, and early dementia [68]. However, longstanding urinary retention requiring chronic CIC might be unavoidable in exceedingly vulnerable elderly patients with CNS lesions and NDO. Moreover, most patients with CNS lesions are vulnerable and cannot handle bladder self-management. As the risk of UTI increases together with PVR after BoNT-A injection, the need for CIC/CISC also increases $[41,69]$. Moreover, patients who develop chronic urinary retention after BoNT-A injection also experience a significant increase in the recovery duration from chronic urinary retention [70]. Hence, patients with CNS lesions who request BoNT-A treatment should be informed of this potential consequence [68].

Patient selection for BoNT-A injection is important considering that those with CNS lesions usually have severe physical 
or mental impairment. Given that bladder fullness and urinary urgency might be impaired after BoNT-A injection, chronic urinary retention and subsequent UTI might develop. As such, an indwelling Foley catheter may be necessary when urinary retention or large PVR volume occurs after BoNT-A injection to avoid bladder overdistention owing to an inappropriate CIC program and to restore adequate bladder perfusion. Quality of life and satisfaction are usually not affected by the need for CIC in patients who can achieve complete dryness after BoNT-A injection [71]. Thus, before injecting BoNT-A in patients with CNS lesions, health costs, the caregiver burden, and potential AEs should be thoroughly discussed with the patients' families [72].

\section{BOTULINUM TOXIN A FOR THE TREATMENT OF PEDIATRIC NEUROGENIC LOWER URINARY TRACT DYSFUNCTION}

BoNT-A has been approved for use in the treatment of urinary incontinence due to NDO or nonneurogenic $\mathrm{OAB}$ with welldocumented treatment outcomes. However, BoNT-A injections have currently not been officially approved for use in pediatric lower urinary tract dysfunction. Urinary incontinence among children is common and needs medical treatment with antimuscarinics or mirabegron. However, if medical treatment fails, BoNT-A injections could be used as an alternative, especially in children with dysfunctional voiding or NDO due to myelomeningocele.

Detrusor BoNT-A injections at a dose of 5 to $10 \mathrm{U} / \mathrm{kg}$ across 30 to 40 sites can increase cystometric bladder capacity, reduce detrusor pressure, and improve bladder compliance in children with myelomeningocele and NDO refractory to antimuscarinic treatment, with the therapeutic duration lasting for 8 months [73-75]. Repeated BoNT-A injections have been found to be as effective as the initial treatment, while patients with vesicoureteral reflux may also benefit from BoNT-A injections [76,77]. Moreover, urethral sphincter BoNT-A injections at a dose of 2 $\mathrm{U} / \mathrm{kg}$ together with detrusor BoNT-A injections at a dose of 8 $\mathrm{U} / \mathrm{kg}$ have been shown to decrease PVR volume, improve urinary incontinence, improve constipation, improve vesicoureteral reflux, and reduce serum creatinine levels among patients with myelomeningocele who had NDO and DSD [78].

Urethral sphincter discoordination during NDO or dysfunctional voiding are highly prevalent in pediatric patients with myelomeningocele or nonneurogenic OAB [79]. Accordingly, urethral sphincter BoNT-A injections at a dose of 50 to $100 \mathrm{U}$ can normalize the flow curve in uroflowmetry and decrease PVR volume among children incapable of emptying their bladder $[80,81]$. One study showed long-term therapeutic efficacy and durability for refractory dysfunctional voiding in children [82].

Current investigations have also demonstrated that detrusor BoNT-A injection is safe and effective for the treatment of pediatric urinary incontinence due to nonneurogenic $\mathrm{OAB}$ refractory to antimuscarinics $[83,84]$. Repeated BoNT-A injections 6 to 7 months after the initial treatment also provided equal clinical and urodynamic effects [85].

\section{DETRUSOR BOTULINUM TOXIN A INJECTIONS AND RENAL FUNCTION IMPROVEMENT IN PATIENTS WITH NEUROGENIC LOWER URINAY TRACT DYSFUNCTION}

Chronic renal failure is a frequently encountered long-term complication in patients with SCI, especially in those with high intravesical pressure and large PVR [86]. Given that detrusor BoNT-A injections can decrease voiding pressure, reduce occurrence of IDCs, and increase bladder compliance, repeated detrusor BoNT-A injections may likely improve renal function in patients with $\mathrm{NDO}$ and upper tract function deterioration. A 6-year follow-up study of patients with SCI who had NDO and received repeated 300-U BoNT-A injections showed that renal pelvis dilatation and vesicoureteral reflux resolved in all patients [37]. However, our short-term study on renal function changes in 33 patients with SCI who received 4 repeated 200-U BoNTA injections revealed no significant improvement in the glomerular filtration rate (GFR) after 2 years [87]. A further comparison of GFR changes between patients with SCI receiving repeated 200- and 300-U BoNT-A injections showed no significant changes between the 300 group and $200 \mathrm{U}$ group at the end-point $(\mathrm{P}=0.197)$. Similarly, no significant difference in GFR changes at the end-point following BoNT-A injections was noted between patients with SCI who had a baseline bladder compliance $\geq 30$ and $<30 \mathrm{~mL} / \mathrm{cm} \mathrm{H}_{2} \mathrm{O}$ in either the 200 or 300 U group [55].

Several reasons may explain why short-term repeated detrusor BoNT-A injections could only maintain renal function, but not improve GFR, in our previous studies, including high intravesical pressure and low renal plasma flow [88-90]. Although detrusor BoNT-A injections can reduce intravesical pressure 
during bladder filling, they might not improve the reduced renal plasma flow in patients with high-level SCI. Therefore, repeated detrusor BoNT-A injections did not improve GFR. Moreover, our study found that IDCs were still present in $89.5 \%$ and $44.1 \%$ of patients with SCI receiving BoNT-A injections of 200 and $300 \mathrm{U} 6$ months after the initial injection, respectively. Thus, detrusor BoNT-A injections may have possibly reduced urinary incontinence, but did not adequately lower intravesical pressure during the storage phase [55]. Adding antimuscarinics or mirabegron might provide additional benefits in reducing detrusor pressure and improving renal function for the long-term bladder management of patients with SCI [91]. Renal function concerns could be an important indication for the long-term use of BoNT-A in patients with NDO who are at high risk for renal function impairment. Numerous modalities can be utilized for bladder management in patients with SCI at high risk for upper urinary tract deterioration. Repeated detrusor BoNT-A injections provide a safe therapeutic modality for not only improving incontinence symptoms, but also preserving renal function.

\section{QUALITY OF LIFE COMPARISON BETWEEN REPEATED DETRUSOR BOTULINUM TOXIN A INJECTIONS AND AUGMENTATION ENTEROCYSTOPLASTY IN PATIENTS WITH SPINAL CORD INJURY}

The therapeutic efficacy of detrusor BoNT-A injections for NDO due to SCI or other CNS lesions has been well established. Repeated BoNT-A injections every 6 to 9 months are necessary for maintaining their therapeutic effects against NDO, especially in patients with chronic SCI. While treatment is generally safe and well tolerated, the most common AEs of detrusor BoNT-A injection include UTIs $[35,45,43]$ and chronic urinary retention $[39,40,56]$, with $\mathrm{AD}$ occasionally occurring during the injection process $[7,29,30]$. Therefore, preoperative antibiotic prophylaxis and postoperative CIC in patients who receive BoNT-A injection are mandatory. Patients with SCI may consider augmentation enterocystoplasty to obtain lifelong therapeutic effects rather than periodical BoNT-A injections, especially when AEs occur after each BoNT-A injection.

Augmentation enterocystoplasty is indicated in patients with reduced bladder capacity and compliance due to NLUTD or other chronic inflammatory bladder diseases $[92,93]$. The procedure has been used to treat bladder dysfunction in adults and pediatric patients with myelomeningocele [94] and can effectively decrease intravesical pressure during bladder storage and increase bladder capacity in patients with end-stage bladder diseases or refractory DO [95]. Although augmentation enterocystoplasty has long-term durability and high satisfaction, discomforting complications still exist [96]. Our recent study of a large cohort of patients with SCI who underwent augmentation enterocystoplasty revealed that $86.9 \%$ of the 76 patients were very satisfied or moderately satisfied with the treatment outcome, while the postoperative incontinence rate was only $16.5 \%$. However, although some patients were able to void spontaneously with the Crede maneuver, $76 \%$ of the patients were completely dependent on CIC. Thus, patients might ultimately opt for an indwelling urethral catheter or cystostomy for convenience of bladder emptying [97].

Considering the high complication rates, augmentation enterocystoplasty is usually reserved as the final treatment modality for NDO in patients with SCI. Currently, urologists suggest detrusor BoNT-A injections for patients with SCI who have NDO or lower bladder compliance, subsequently recommending augmentation enterocystoplasty to reconstruct the diseased bladder and increase bladder capacity only when the NDO is refractory to repeat BoNT-A injections. A previous prospective study that compared the quality of life between a small group of patients who underwent augmentation enterocystoplasty $(n=16)$ or repeated BoNT-A injections $(n=14)$ found that the BoNT-A group had a significantly lower continence rate $(87.5 \%$ vs. $42.3 \%, \mathrm{P}=0.0187$ ), whereas the augmentation enterocystoplasty group had a significantly higher quality of life index using the Qualiveen-30 instrument (1.625 vs. $1.077, \mathrm{P}=0.037)$. None of the patients receiving augmentation enterocystoplasty had low bladder compliance or a higher detrusor pressure at bladder capacity [33].

While detrusor BoNT-A injections can be considered as a medical procedure for bladder augmentation, augmentation enterocystoplasty can be rationally recommended as the final form of bladder management when treatment is ineffective or frequent detrusor injections are intolerable.

\section{PATIENT SATISFACTION WITH DETRUSOR OR URETHRAL BOTULINUM TOXIN A INJECTION FOR NEUROGENIC LOWER URINARY TRACT DYSFUNCTION}

Appropriate management of lower urinary tract dysfunction in 
patients with SCI can be challenging to urologists. One study showed that most patients with suprasacral SCI exhibited NDO with or without DSD [98]. Bladder self-management by patients relies on good hand dexterity, strong abdominal muscles, intact bladder sensation, and urethral sphincter coordination during voiding stimulation [98]. Currently, detrusor BoNT-A injections to decrease detrusor contractility [6,7], urethral sphincter BoNT-A injections to reduce urethral resistance [2,5], or combined detrusor and urethral BoNT-A injections to simultaneously improve bladder storage and emptying can be utilized [99].

Repeated BoNT-A injections for the treatment of NDO were strongly recommended by a European consensus report [72] Another study recommended that BoNT-A retreatment be administered over an interval of 6 to 9 months [16]. As such, patients who do not respond well to the first BoNT-A injection still need adequate time for retreatment. The feasibility of BoNT-A injection into the detrusor or urethral sphincter of patients with SCI exhibiting NDO and DSD depends on the patients' will and bladder condition. Accordingly, detrusor BoNTA injection should be performed in patients who have a contracted bladder with low compliance to protect the upper urinary tract function. After detrusor BoNT-A injection, most patients expressed satisfaction with quality of life despite the need for CIC. However, patients who opted for urethral BoNT-A injections for DSD treatment might experience bothersome incontinence, while still exhibiting incomplete bladder emptying, prompting the need for CIC for periodic bladder emptying. Our previous study showed that patients with NDO and DSD who received detrusor BoNT-A injections had greater quality of life improvements than those receiving urethral BoNT-A injections [23]. However, a discrepancy was observed between objective urodynamic outcomes and actual patient satisfaction in the treatment of DSD by urethral or detrusor BoNT-A injections.

The adherence rates in patients with SCI receiving detrusor BoNT-A injections depend on treatment outcomes, with those receiving BoNT-A injections for NDO generally having high satisfaction rates [48]. Recurrent UTI, AD during injection, CIC-related issues, and persistent urinary incontinence are the primary causes for discontinuation of BoNT-A. Some patients have been converted to augmentation enterocystoplasty following repeated detrusor BoNT-A injections after expressing their desire to have a permanent correction without periodic injections. Nonetheless, patients with SCI who have minor compli- cations after BoNT-A injections can usually tolerate complications and continue with detrusor BoNT-A injections when the therapeutic efficacy satisfies their expectations.

\section{CONCLUSIONS}

BoNT-A injections have been widely utilized for the treatment of NLUTD and LUTD in patients with SCI and those with other neurogenic lesions, such as MS, CVA, PD, dementia, and pediatric NLUTD. Treatment outcomes have been associated with the patients' lower urinary tract condition and their preference for bladder management. However, some AEs after BoNT-A injections, such as UTI, large PVR volume, and urinary retention requiring $\mathrm{CIC}$, are still important concerns that need careful management. As such, complete informed consent for BoNT-A injection therapy with full disclosure of its potential complications should be obtained before this procedure is undertaken for any patient with NLUTD.

\section{AUTHOR CONTRIBUTION STATEMENT}

- Conceptualization: $\mathrm{HCK}$

-Writing-original draft: YHJ, SFC, HCK

- Writing-review \& editing: $H C K$

\section{REFERENCES}

1. Dykstra DD, Sidi AA, Scott AB, Pagel JM, Goldish GD. Effects of botulinum A toxin on detrusor-sphincter dyssynergia in spinal cord injury patients. J Urol 1988;139:919-22.

2. Schurch B, Hauri D, Rodic B, Curt A, Meyer M, Rossier AB. Botulinum-A toxin as a treatment of detrusor-sphincter dyssynergia: a prospective study in 24 spinal cord injury patients. J Urol 1996; 155:1023-9.

3. Dykstra DD, Sidi AA. Treatment of detrusor-sphincter dyssynergia with botulinum A toxin: a double-blind study. Arch Phys Med Rehabil 1990;71:24-6.

4. de Sèze M, Petit H, Gallien P, de Sèze MP, Joseph PA, Mazaux JM, et al. Botulinum a toxin and detrusor sphincter dyssynergia: a double-blind lidocaine-controlled study in 13 patients with spinal cord disease. Eur Urol 2002;42:56-62.

5. Kuo HC. Botulinum A toxin urethral injection for the treatment of lower urinary tract dysfunction. J Urol 2003;170:1908-12.

6. Schurch B, Stöhrer M, Kramer G, Schmid DM, Gaul G, Hauri D. Botulinum-A toxin for treating detrusor hyperreflexia in spinal 
cord injured patients: a new alternative to anticholinergic drugs? Preliminary results. J Urol 2000;164:692-7.

7. Reitz A, Stöhrer M, Kramer G, Del Popolo GD, Chartier-Kastler E, Pannek J, et al. European experience of 200 cases treated with botulinum-A toxin injections into the detrusor muscle for urinary incontinence due to neurogenic detrusor overactivity. Eur Urol 2004;45:510-5.

8. Dong M, Yeh F, Tepp WH, Dean C, Johnson EA, Janz R, et al. SV2 is the protein receptor for botulinum neurotoxin A. Science 2006; 312:592-6.

9. Cruz F. Targets for botulinum toxin in the lower urinary tract. Neurourol Urodyn 2014;33:31-8.

10. Kanai A, Zabbarova I, Oefelein M, Radziszewski P, Ikeda Y, Anderssonet KE. Mechanisms of action of botulinum neurotoxins, beta3adrenergic receptor agonists, and PDE5 inhibitors in modulating detrusor function in overactive bladders: ICI-RS 2011. Neurourol Urodyn 2012;31:300-8.

11. Apostolidis A, Dasgupta P, Fowler CJ. Proposed mechanism for the efficacy of injected botulinum toxin in the treatment of human detrusor overactivity. Eur Urol 2006;49:644-50.

12. Schulte-Baukloh H, Priefert J, Knispel HH, Lawrence GW, Miller K, Neuhaus J. Botulinum toxin A detrusor injections reduce postsynaptic muscular M2, M3, P2X2, and P2X3 receptors in children and adolescents who have neurogenic detrusor overactivity: a singleblind study. Urology 2013;81:1052-7.

13. Smith CP, Vemulakonda VM, Kiss S, Boone TB, Somogyi GT. Enhanced ATP release from rat bladder urothelium during chronic bladder inflammation: effect of botulinum toxin A. Neurochem Int 2005;47:291-7.

14. Smith CP, Gangitano DA, Munoz A, Salas NA, Boone TB, Aoki $\mathrm{KR}$, et al. Botulinum toxin type A normalizes alterations in urothelial ATP and NO release induced by chronic spinal cord injury. Neurochem Int 2008;52:1068-75.

15. Yiangou Y, Facer P, Ford A, Brady C, Wiseman O, Fowler CJ, et al. Capsaicin receptor VR1 and ATP-gated ion channel P2X3 in human urinary bladder. BJU Int 2001;87:774-9.

16. Schurch B, de Sèze M, Denys P, Chartier-Kastler E, Haab F, Everaert $\mathrm{K}$, et al. Botulinum toxin type $\mathrm{A}$ is a safe and effective treatment for neurogenic urinary incontinence: results of a single treatment, randomized, placebo controlled 6-month study. J Urol 2005;174:196-200.

17. Phelan MW, Franks M, Somogyi GT, Yokoyama T, Fraser MO, Lavelle JP, et al. Botulinum toxin urethral sphincter injection to restore bladder emptying in men and women with voiding dysfunction. J Urol 2001;165:1107-10.
18. Mehta S, Hill D, Foley N, Hsieh J, Ethans K, Potter P, et al. A metaanalysis of botulinum toxin sphincteric injections in the treatment of incomplete voiding after spinal cord injury. Arch Phys Med Rehabil 2012;93:597-603.

19. Kuo HC. Satisfaction with urethral injection of botulinum toxin A for detrusor sphincter dyssynergia in patients with spinal cord lesion. Neurourol Urodyn 2008;27:793-6.

20. Smith CP, Nishiguchi J, O’Leary M, Yoshimura N, Chancellor MB. Single-institution experience in 110 patients with botulinum toxin A injection into bladder or urethra. Urology 2005;65:37-41.

21. Gallien P, Reymann JM, Amarenco G, Nicolas B, de Sèze M, Bellissant E. Placebo controlled, randomised, double blind study of the effects of botulinum A toxin on detrusor sphincter dyssynergia in multiple sclerosis patients. J Neurol Neurosurg Psychiatry 2005;76:1670-6.

22. Mahfouz W, Karsenty G, Corcos J. Injection of botulinum toxin type A in the urethral sphincter to treat lower urinary tract dysfunction: review of indications, techniques and results: 2011 update. Can J Urol 2011;18:5787-95.

23. Kuo HC. Therapeutic outcome and quality of life between urethral and detrusor botulinum toxin treatment for patients with spinal cord lesions and detrusor sphincter dyssynergia. Int J Clin Pract 2013;67:1044-9.

24. Chen YH, Kuo HC. Botulinum A toxin treatment of urethral sphincter pseudodyssynergia in patients with cerebrovascular accidents or intracranial lesions. Urol Int 2004;73:156-61.

25. Vallès M, Benito J, Portell E, Vidal J. Cerebral hemorrhage due to autonomic dysreflexia in a spinal cord injury patient. Spinal Cord 2005;43:738-40.

26. Karlsson AK. Autonomic dysreflexia. Spinal Cord 1999;37:383-91.

27. Tsai SJ, Ying TH, Huang YH, Cheng JW, Bih LI, Lew HL. Transperineal injection of botulinum toxin A for treatment of detrusor sphincter dyssynergia: localization with combined fluoroscopic and electromyographic guidance. Arch Phys Med Rehabil 2009; 90:832-6.

28. Michel F, Ciceron C, Bernuz B, Boissier R, Gaillet S, Even A, et al. Botulinum toxin type A injection after failure of augmentation enterocystoplasty performed for neurogenic detrusor overactivity: preliminary results of a salvage strategy. The ENTEROTOX Study. Urology 2019;129:43-7.

29. Tow AM, Toh KL, Chan SP, Consigliere D. Botulinum toxin type A for refractory neurogenic detrusor overactivity in spinal cord injured patients in Singapore. Ann Acad Med Singapore 2007;36:117.

30. Chen SF, Kuo HC. Therapeutic outcome and patient adherence to 
repeated onabotulinumtoxinA detrusor injections in chronic spinal cord-injured patients and neurogenic detrusor overactivity. J Formos Med Assoc 2015;114:583-9.

31. Ehren I, Volz D, Farrelly E, Berglund L, Brundin L, Hultling C, et al. Efficacy and impact of botulinum toxin $\mathrm{A}$ on quality of life in patients with neurogenic detrusor overactivity: a randomized, placebo-controlled, double-blind study. Scand J Urol Nephrol 2007;41: 335-40.

32. Patki PS, Hamid R, Arumugam K, Shah PJ, Craggs M. Botulinum toxin-type $\mathrm{A}$ in the treatment of drug-resistant neurogenic detrusor overactivity secondary to traumatic spinal cord injury. BJU Int 2006;98:77-82.

33. Anquetil C, Abdelhamid S, Gelis A, Fattal C. Botulinum toxin therapy for neurogenic detrusor hyperactivity versus augmentation enterocystoplasty: impact on the quality of life of patients with SCI. Spinal Cord 2016;54:1031-5.

34. Kennelly M, Dmochowski R, Ethans K, Karsenty G, Schulte-Baukloh $\mathrm{H}$, Jenkins B, et al. Long-term efficacy and safety of onabotulinumtoxinA in patients with urinary incontinence due to neurogenic detrusor overactivity: an interim analysis. Urology 2013;81: 491-7.

35. Rovner E, Dmochowski R, Chapple C, Thompson C, Lam W, Haag-Molkenteller C. OnabotulinumtoxinA improves urodynamic outcomes in patients with neurogenic detrusor overactivity. Neurourol Urodyn 2013;32:1109-15.

36. Apostolidis A, Thompson C, Yan X, Mourad S. An exploratory, placebo-controlled, dose-response study of the efficacy and safety of onabotulinumtoxinA in spinal cord injury patients with urinary incontinence due to neurogenic detrusor overactivity. World J Urol 2013;31:1469-74.

37. Giannantoni A, Mearini E, Del Zingaro M, Porena M. Six-year follow-up of botulinum toxin A intradetrusorial injections in patients with refractory neurogenic detrusor overactivity: clinical and urodynamic results. Eur Urol 2009;55:705-11.

38. Kalsi V, Apostolidis A, Popat R, Gonzales G, Fowler CJ, Dasgupta P. Quality of life changes in patients with neurogenic versus idiopathic detrusor overactivity after intradetrusor injections of botulinum neurotoxin type A and correlations with lower urinary tract symptoms and urodynamic changes. Eur Urol 2006;49:528-35.

39. Cruz F, Herschorn S, Aliotta P, Brin M, Thompson C, Lam W, et al. Efficacy and safety of onabotulinumtoxinA in patients with urinary incontinence due to neurogenic detrusor overactivity: a randomized, double-blind, placebo-controlled trial. Eur Urol 2011;60:74250 .

40. Akbar M, Abel R, Seyler TM, Bedke J, Haferkamp A, Gerner HJ, et al. Repeated botulinum-A toxin injections in the treatment of myelodysplastic children and patients with spinal cord injuries with neurogenic bladder dysfunction. BJU Int 2007;100:639-45.

41. Mangera A, Apostolidis A, Andersson KE, Dasgupta P, Giannantoni A, Roehrborn C, et al. An updated systematic review and statistical comparison of standardised mean outcomes for the use of botulinum toxin in the management of lower urinary tract disorders. Eur Urol 2014;65:981-90.

42. Schurch B, Denys P, Kozma CM, Reese PR, Slaton T, Barron RL. Botulinum toxin A improves the quality of life of patients with neurogenic urinary incontinence. Eur Urol 2007;52:850-58.

43. Gamé X, Castel-Lacanal E, Bentaleb Y, Thiry-Escudié I, De Boissezon XD, Malavaud B, et al. Botulinum toxin A detrusor injections in patients with neurogenic detrusor overactivity significantly decrease the incidence of symptomatic urinary tract infections. Eur Urol 2008;53:613-8.

44. MacDonald R, Fink HA, Huckabay C, Monga M, Wilt TJ. Botulinum toxin for treatment of urinary incontinence due to detrusor overactivity: a systematic review of effectiveness and adverse effects. Spinal Cord 2007;45:535-41.

45. Mouttalib S, Khan S, Castel-Lacanal E, Guillotreau J, De Boissezon $\mathrm{XD}$, Malavaud B, et al. Risk of urinary tract infection after detrusor botulinum toxin A injections for refractory neurogenic detrusor overactivity in patients with no antibiotic treatment. BJU Int 2010;106:1677-80.

46. Chen CY, Liao CH, Kuo HC. Therapeutic effects of detrusor botulinum toxin A injection on neurogenic detrusor overactivity in patients with different levels of spinal cord injury and types of detrusor sphincter dyssynergia. Spinal Cord 2011;49:659-64.

47. Ginsberg D, Cruz F, Herschorn S, Gousse A, Keppenne V, Aliotta P, et al. OnabotulinumtoxinA is effective in patients with urinary incontinence due to neurogenic detrusor overactivity [corrected] regardless of concomitant anticholinergic use or neurologic etiology. Adv Ther 2013;30:819-33.

48. Hori S, Patki P, Attar KH, Ismail S, Vasconcelos JC, Shah PJ. Patients' perspective of botulinum toxin-A as a long-term treatment option for neurogenic detrusor overactivity secondary to spinal cord injury. BJU Int 2009;104:216-20.

49. Chancellor MB, Patel V, Leng WW, Shenot PJ, Lam W, Globe DR, et al. OnabotulinumtoxinA improves quality of life in patients with neurogenic detrusor overactivity. Neurology 2013;81:841-8.

50. Mangera A, Andersson KE, Apostolidis A, Chapple C, Dasgupta P, Giannantoni A, et al. Contemporary management of lower urinary tract disease with botulinum toxin A: a systematic review of Botox (onabotulinumtoxinA) and Dysport (abobotulinumtoxinA). Eur 
Urol 2011;60:784-95.

51. Kuo HC. Urodynamic evidence of effectiveness of botulinum A toxin injection in treatment of detrusor overactivity refractory to anticholinergic agents. Urology 2004;63:868-72.

52. Ginsberg D, Gousse A, Keppenne V, Sievert KD, Thompson C, Lam W, et al. Phase 3 efficacy and tolerability study of onabotulinumtoxinA for urinary incontinence from neurogenic detrusor overactivity. J Urol 2012;187:2131-9.

53. Herschorn S, Gajewski J, Ethans K, Corcos J, Carlson K, Bailly G, et al. Efficacy of botulinum toxin A injection for neurogenic detrusor overactivity and urinary incontinence: a randomized, double-blind trial. J Urol 2011;185:2229-35.

54. Sussman D, Patel V, Del Popolo G, Lam W, Globe D, Pommerville P. Treatment satisfaction and improvement in health-related quality of life with onabotulinumtoxinA in patients with urinary incontinence due to neurogenic detrusor overactivity. Neurourol Urodyn 2013;32:242-9.

55. Chen YC, Kuo HC. The therapeutic effects of repeated detrusor injections between 200 or 300 units of onabotulinumtoxinA in chronic spinal cord injured patients. Neurourol Urodyn 2014;33: 129-34

56. Kuo HC. Therapeutic satisfaction and dissatisfaction in patients with spinal cord lesions and detrusor sphincter dyssynergia who received detrusor botulinum toxin a injection. Urology 2008;72: 1056-60.

57. McKenzie P, Badlani GH. The incidence and etiology of overactive bladder in patients after cerebrovascular accident. Curr Urol Rep 2012;13:402-6.

58. Ruffion A, Castro-Diaz D, Patel H, Khalaf K, Onyenwenyi A, Globe D, et al. Systematic review of the epidemiology of urinary incontinence and detrusor overactivity among patients with neurogenic overactive bladder. Neuroepidemiology 2013;41:146-55.

59. Schulte-Baukloh H, Schobert J, Stolze T, Stürzebecher B, Weiss C, Knispel HH. Efficacy of botulinum-A toxin bladder injections for the treatment of neurogenic detrusor overactivity in multiple sclerosis patients: an objective and subjective analysis. Neurourol Urodyn 2006;25:110-5.

60. Khan S, Game X, Kalsi V, Gonzales G, Panicker J, Elneil S, et al. Long-term effect on quality of life of repeat detrusor injections of botulinum neurotoxin-A for detrusor overactivity in patients with multiple sclerosis. J Urol 2011;185:1344-9.

61. Gaillet S, Bardot P, Bernuz B, Boissier R, Lenne-Aurier K, ThiryEscudier I, et al. Five years follow-up study and failures analysis of botulinum toxin repeated injections to treat neurogenic detrusor overactivity. Prog Urol 2012;22:1064-70.
62. Mehnert U, Birzele J, Reuter K, Schurch B. The effect of botulinum toxin type A on overactive bladder symptoms in patients with multiple sclerosis: a pilot study. J Urol 2010;184:1011-6.

63. Kulaksizoglu H, Parman Y. Use of botulinim toxin-A for the treatment of overactive bladder symptoms in patients with Parkinsons's disease. Parkinsonism Relat Disord 2010;16:531-4.

64. Giannantoni A, Conte A, Proietti S, Giovannozzi S, Rossi A, Fabbrini $\mathrm{G}$, et al. Botulinum toxin type A in patients with Parkinson's disease and refractory overactive bladder. J Urol 2011;186:960-4.

65. Giannantoni A, Rossi A, Mearini E, Del Zingaro MD, Porena M, Berardelli A. Botulinum toxin A for overactive bladder and detrusor muscle overactivity in patients with Parkinson's disease and multiple system atrophy. J Urol 2009;182:1453-7.

66. Anderson RU, Orenberg EK, Glowe P. OnabotulinumtoxinA office treatment for neurogenic bladder incontinence in Parkinson's disease. Urology 2014;83:22-7.

67. Kuo HC. Therapeutic effects of suburothelial injection of botulinum a toxin for neurogenic detrusor overactivity due to chronic cerebrovascular accident and spinal cord lesions. Urology 2006;67: 232-6.

68. Jiang YH, Liao CH, Tang DL, Kuo HC. Efficacy and safety of intravesical onabotulinumtoxinA injection on elderly patients with chronic central nervous system lesions and overactive bladder. PLoS One 2014;9:e105989.

69. Dmochowski R, Chapple C, Nitti VW, Chancellor MB, Everaert K, Thompson C, et al. Efficacy and safety of onabotulinumtoxinA for idiopathic overactive bladder: a double-blind, placebo controlled, randomized, dose ranging trial. J Urol 2010;184:2416-22.

70. Liao $\mathrm{CH}$, Kuo HC. Increased risk of large post-void residual urine and decreased long-term success rate after intravesical onabotulinumtoxinA injection for refractory idiopathic detrusor overactivity. J Urol 2013;189:1804-10.

71. Schurch B, Carda S. OnabotulinumtoxinA and multiple sclerosis. Ann Phys Rehabil Med 2014;57:302-14.

72. Apostolidis A, Dasgupta P, Denys P, Elneil S, Fowler CJ, Giannantoni $\mathrm{A}$, et al. Recommendations on the use of botulinum toxin in the treatment of lower urinary tract disorders and pelvic floor dysfunctions: a European consensus report. Eur Urol 2009;55:100-19.

73. Schulte-Baukloh H, Michael T, Schobert J, Stolze T, Knispel HH. Efficacy of botulinum-a toxin in children with detrusor hyperreflexia due to myelomeningocele: preliminary results. Urology 2002; 59:325-7.

74. Riccabona M, Koen M, Schindler M, Goedele B, Pycha A, Lusuardi $\mathrm{L}$, et al. Botulinum-A toxin injection into the detrusor: a safe alternative in the treatment of children with myelomeningocele with 
detrusor hyperreflexia. J Urol 2004;171:845-8.

75. Altaweel W, Jednack R, Bilodeau C, Corcos J. Repeated intradetrusor botulinum toxin type A in children with neurogenic bladder due to myelomeningocele. J Urol 2006;175:1102-5.

76. Kajbafzadeh AM, Moosavi S, Tajik P, Arshadi H, Payabvash S, Salmasi AH, et al. Intravesical injection of botulinum toxin type A: management of neuropathic bladder and bowel dysfunction in children with myelomeningocele. Urology 2006;68:1091-6.

77. Horst M, Weber DM, Bodmer C, Gobet R. Repeated botulinum-A toxin injection in the treatment of neuropathic bladder dysfunction and poor bladder compliance in children with myelomeningocele. Neurourol Urodyn 2011;30:1546-49.

78. Safari S, Jamali S, Habibollahi P, Arshadi H, Nejat F, Kajbafzadeh AM. Intravesical injections of botulinum toxin type A for management of neuropathic bladder: a comparison of two methods. Urology 2010;76:225-30.

79. Kuo HC, Liu HT. Investigation of dysfunctional voiding in children with urgency frequency syndrome and urinary incontinence. Urol Int 2006;76:72-6.

80. Radojicic ZI, Perovic SV, Milic NM. Is it reasonable to treat refractory voiding dysfunction in children with botulinum-A toxin? J Urol 2006;176:332-6.

81. Mokhless I, Gaafar S, Fouda K, Shafik M, Assem A. Botulinum A toxin urethral sphincter injection in children with nonneurogenic neurogenic bladder. J Urol 2006;176:1767-70.

82. Vricella GJ, Campigotto M, Coplen DE, Traxel EJ, Austin PF. Longterm efficacy and durability of botulinum-A toxin for refractory dysfunctional voiding in children. J Urol 2014;191:1586-91.

83. Lahdes-Vasama TT, Anttila A, Wahl E, Taskinen S. Urodynamic assessment of children treated with botulinum toxin A injections for urge incontinence: a pilot study. Scand J Urol Nephrol 2011;45: 397-400.

84. McDowell DT, Noone D, Tareen F, Waldron M, Quinn F. Urinary incontinence in children: botulinum toxin is a safe and effective treatment option. Pediatr Surg Int 2012;28:315-20.

85. Marte A, Borrelli M, Sabatino MD, Balzo BD, Prezioso M, Pintozzi $\mathrm{L}$, et al. Effectiveness of botulinum-A toxin for the treatment of refractory overactive bladder in children. Eur J Pediatr Surg 2010;20: 153-7.

86. El-Masri WS, Chong T, Kyriakider AE, Wang D. Long-term followup study of outcomes of bladder management in spinal cord injury patients under the care of the Midlands Centre for spinal injury in
Oswestry. Spinal Cord 2012;50:14-21.

87. Kuo HC, Liu SH. Effect of repeated detrusor onabotulinumtoxinA injections on bladder and renal function in patients with chronic spinal cord injuries. Neurourol Urodyn 2011;30:1541-5.

88. Jacobson AF, Britell C, Little JW. Relation of changes in radionuclide measures of effective renal plasma flow and glomerular filtration rate in spinal cord injury patients. J Am Paraplegia Soc 1994; 17:15-9.

89. Just A, Ehmke H, Wittmann U, Kirchheim HR. Role of angiotensin II in dynamic renal blood flow autoregulation of the conscious dog. J Physiol 2002;538:167-77.

90. Cameron AP, Rodriguez GM, Schomer KG. Systematic review of urological followup after spinal cord injury. J Urol 2012;187:391-7.

91. Krebs J, Pannek J, Rademacher F, Wöllner J. Real-world effects of mirabegron in patients with chronic neurogenic detrusor overactivity - a retrospective cohort study. Res Rep Urol 2020;12:187-92.

92. Chen JL, Kuo HC. Long-term outcomes of augmentation enterocystoplasty with an ileal segment in patients with spinal cord injury. J Formos Med Assoc 2009;108:475-80.

93. Krebs J, Bartel P, Pannek J. Functional outcome of supratrigonal cystectomy and augmentation ileocystoplasty in adult patients with refractory neurogenic lower urinary tract dysfunction. Neurourol Urodyn 2016;35:260-6.

94. Lendvay TS, Cowan CA, Mitchell MM, Joyner BD, Grady RW. Augmentation cystoplasty rates at children's hospitals in the United States: a Pediatric Health Information System database study. J Urol 2006;176:1716-20.

95. Reyblat P, Ginsberg DA. Augmentation cystoplasty: what are the indications? Curr Urol Rep 2008;9:452-8.

96. $\mathrm{Ku} \mathrm{JH}$. The management of neurogenic bladder and quality of life in spinal cord injury. BJU Int 2006;98:739-45.

97. Wu SY, Kuo HC. A real-world experience with augmentation enterocystoplasty-High patient satisfaction with high complication rates. Neurourol Urodyn 2018;37:744-50.

98. Weld KJ, Dmochowski RR. Association of Level of injury and bladder behavior in patients with post-traumatic spinal cord injury. Urology 2000;55:490-4.

99. Schulte-Baukloh H, Weiss C, Stolze T, Herholz J, Stürzebecher B, Miller K, et al. Botulinum-A toxin detrusor and sphincter injection in treatment of overactive bladder syndrome: objective outcome and patient satisfaction. Eur Urol 2005;48:984-90. 